\title{
Simultaneous diagnosis of radial profiles and mix in NIF ignition-scale implosions via $\mathrm{X}$-ray spectroscopy
}

O. Ciricosta, H. Scott, P. Durey, B. A. Hammel, R. Epstein, T. R. Preston, S. P. Regan, S. M. Vinko, N. C. Woolsey, and J. S. Wark

Citation: Physics of Plasmas 24, 112703 (2017); doi: 10.1063/1.5000774

View online: https://doi.org/10.1063/1.5000774

View Table of Contents: http://aip.scitation.org/toc/php/24/11

Published by the American Institute of Physics

\section{Articles you may be interested in}

A comprehensive alpha-heating model for inertial confinement fusion

Physics of Plasmas 25, 012703 (2018); 10.1063/1.4991405

On the importance of minimizing "coast-time" in x-ray driven inertially confined fusion implosions

Physics of Plasmas 24, 092706 (2017); 10.1063/1.4994856

Laser-driven magnetized liner inertial fusion

Physics of Plasmas 24, 062701 (2017); 10.1063/1.4984779

The physics of long- and intermediate-wavelength asymmetries of the hot spot: Compression hydrodynamics and energetics

Physics of Plasmas 24, 102704 (2017); 10.1063/1.4995250

Capsule physics comparison of National Ignition Facility implosion designs using plastic, high density carbon, and beryllium ablators

Physics of Plasmas 25, 032703 (2018); 10.1063/1.5016874

Improving ICF implosion performance with alternative capsule supports

Physics of Plasmas 24, 056302 (2017); 10.1063/1.4977536

\section{PHYSICS TODAY}

MANAGER'S GUIDE

WHITEPAPERS
READ NOW

PRESENTED BY

Accelerate R\&D with

Multiphysics Simulation 


\title{
Simultaneous diagnosis of radial profiles and mix in NIF ignition-scale implosions via X-ray spectroscopy
}

\author{
O. Ciricosta, ${ }^{1}$ H. Scott, ${ }^{2}$ P. Durey, ${ }^{3}$ B. A. Hammel, ${ }^{2}$ R. Epstein, ${ }^{4}$ T. R. Preston, ${ }^{1}$ S. P. Regan, ${ }^{4}$ \\ S. M. Vinko, ${ }^{1}$ N. C. Woolsey, ${ }^{3}$ and J. S. Wark ${ }^{1, a)}$ \\ ${ }^{1}$ Department of Physics, Clarendon Laboratory, University of Oxford, Parks Road, Oxford OXI 3PU, \\ United Kingdom \\ ${ }^{2}$ Lawrence Livermore National Laboratory, Livermore, California 94550, USA \\ ${ }^{3}$ Department of Physics, University of York, York YO10 5DD, United Kingdom \\ ${ }^{4}$ Laboratory for Laser Energetics, University of Rochester, Rochester, New York 14623-1299, USA
}

(Received 18 August 2017; accepted 4 October 2017; published online 6 November 2017)

\begin{abstract}
In a National Ignition Facility implosion, hydrodynamic instabilities may cause the cold material from the imploding shell to be injected into the hot-spot (hot-spot mix), enhancing the radiative and conductive losses, which in turn may lead to a quenching of the ignition process. The boundbound features of the spectrum emitted by high-Z ablator dopants that get mixed into the hot-spot have been previously used to infer the total amount of mixed mass; however, the typical errorbars are larger than the maximum tolerable mix. We present here an improved 2D model for mix spectroscopy which can be used to retrieve information on both the amount of mixed mass and the full imploded plasma profile. By performing radiation transfer and simultaneously fitting all of the features exhibited by the spectra, we are able to constrain self-consistently the effect of the opacity of the external layers of the target on the emission, thus improving the accuracy of the inferred mixed mass. The model's predictive capabilities are first validated by fitting simulated spectra arising from fully characterized hydrodynamic simulations, and then, the model is applied to previously published experimental results, providing values of mix mass in agreement with previous estimates. We show that the new self consistent procedure leads to better constrained estimates of mix and also provides insight into the sensitivity of the hot-spot spectroscopy to the spatial properties of the imploded capsule, such as the in-flight aspect ratio of the cold fuel surrounding the hotspot. Published by AIP Publishing. https://doi.org/10.1063/1.5000774
\end{abstract}

\section{INTRODUCTION}

The formation of a stable central hotspot with sufficiently high temperature is one of the key requirements for an inertial confinement fusion (ICF) implosion to achieve ignition. ${ }^{1}$ One of the factors that may prevent this occurring is the ability of hydrodynamic instabilities to inject the cold material-emanating from the external layers of the capsule-into the hotspot ${ }^{2}$ (hotspot mix): with a typical ICF design, as used at the National Ignition Facility (NIF) during the National Ignition Campaign ${ }^{3,4}$ (NIC), a mix mass just above $75 \mathrm{ng}$ contaminating a hotspot of $\sim 2 \times 10^{4} \mathrm{ng}$ is estimated to be large enough to significantly degrade the implosion efficiency via radiative cooling. ${ }^{5}$ Indeed, a sharp drop in the neutron yield is observed for shots with an estimated mix mass above this limit. ${ }^{6}$ It is thus of considerable interest to develop diagnostic tools that would allow the measurement of low amounts of mix mass in ICF implosions, preferably with an accuracy better than the $75 \mathrm{ng}$ limit.

Spectroscopic techniques, originally developed to diagnose the core conditions in compressed micro-balloons, ${ }^{7-9}$ have been used over the past few years to estimate the level of mix in NIF implosions. In this first approach to the problem, an analysis of the $\mathrm{Ge} \mathrm{He}_{\alpha}$ emission resulting from mix in implosions with a Ge-doped plastic ablator was

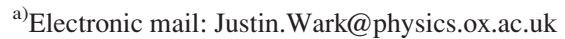

undertaken. ${ }^{6,10}$ This technique, although not able to detect mix from undoped regions of the shell nor mix mass that is too cold to emit $\mathrm{He}_{\alpha}$ emission, is sensitive to low levels of mix $(<75 \mathrm{ng})$ and was also able to reveal (by using multidoped targets) that the instabilities leading to mix are mainly located at the ablation front. ${ }^{6}$ A second technique, based on the enhancement of the continuum emission relative to the neutron yield, ${ }^{11}$ does not rely on the presence of high-Z dopants in the mix: this makes it suitable for recent implosion campaigns where Ge dopants have been replaced with Si dopants (resulting in improved implosion performance, but no line emission available for mix spectroscopy), but also makes it relatively insensitive to low levels of mix ( $\$ 150 \mathrm{ng}$ ).

Unfortunately, both spectroscopic techniques rely on an estimate of the optical thickness of the compressed shellwhich limits the accuracy of the mix measurement-and both provide values for the mix mass with errorbars larger than the aforementioned radiative cooling limit of $75 \mathrm{ng}$. However, the Ge spectroscopy spectra acquired thus far contain several other features in addition to the $\mathrm{He}_{\alpha}$ profiles that can be used to infer quantities like the shell opacity. ${ }^{10} \mathrm{We}$ present here a 2D model which improves the previous analysis of the Ge spectra by self-consistently fitting all such spectral features at once (bound-bound, bound-free, and freefree), thus providing a better constrained estimate of mix. We show that the improved accuracy is better than the $75 \mathrm{ng}$ limit for Ge implosions with a low level of mix and that the 
self-consistent analysis is also sensitive to the radial plasma profile of the imploded capsule. Although the focus here will be on analysing implosions with a Ge-doped plastic shell (or a shell doped with similar high-Z elements), which provides the best estimate for low levels of mix mass, the results inferred from the bound-free and free-free features of the spectra also apply to the study of more general implosions: the model is thus intended to be a useful tool even for the spectroscopic diagnosis of more recent ICF shots with Sidoped capsules or for the analysis and design of non-ignition experiments based on spectroscopy, such as self-radiography implosions. $^{12}$

The remainder of this paper is divided as follows: Sec. II describes the 2D model; in Sec. III, the performance of the model is tested by fitting synthetic spectra from hydrodynamic simulations and comparing the resulting plasma profiles; in Sec. IV, the model is then applied to diagnose mix for previously published experimental data ${ }^{6}$ and its diagnostic abilities are discussed; in Sec. V, we draw the conclusions.

\section{2D CRETIN MODEL}

In order to model the emission spectra, including all of the main spectral features observed in the experiment, we have built a $2 \mathrm{D}$ representation of an imploded ICF target and of the radiation transfer between its layers by using the simulation code Cretin. ${ }^{13,14}$ The geometry of the model is depicted in Fig. 1, whilst a typical simulated spectrum is shown in Fig. 2. We first describe here the geometry of the

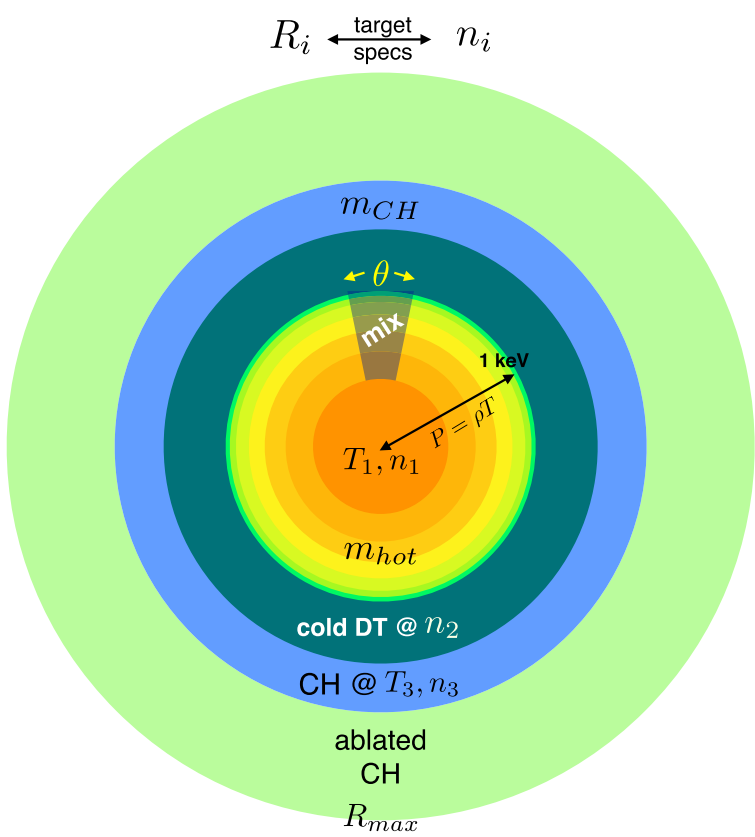

FIG. 1. Schematic diagram of the target geometry used to model the mix spectra. The radii of the different layers in each simulation are constrained by the ion densities (used as fit parameters) and by the known amount of each material (by experimental design). Radiation transfer is performed through the different layers, and then a set of rays parallel to the equator (i.e., perpendicular to the mix jet) is used to compute the emitted spectral intensity seen by an equatorial spectrometer. The predicted signal is calculated assuming constant conditions through the X-ray burnwidth time (125 ps).

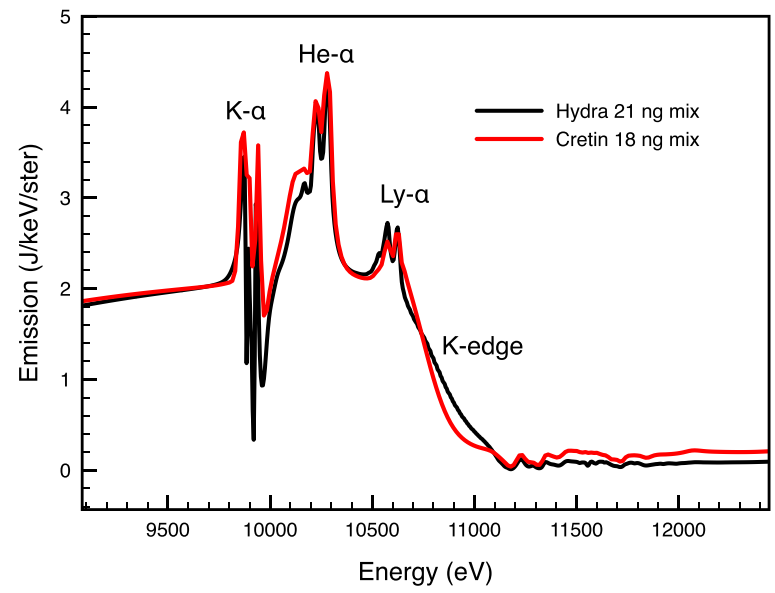

FIG. 2. Emission spectrum produced by postprocessing a hydrodynamic simulation (Hydra) of an implosion where a jet containing $21 \mathrm{ng}$ of $\mathrm{Ge}: \mathrm{CH}$ is driven into the hotspot by instabilities, compared with a fit obtained by using our 2D model (Cretin).

simulated target and will then describe how the different regions relate to the spectral features in Sec. III.

The idealized imploded capsule in Fig. 1 can be roughly divided into three main regions, proceeding from the center outwards:

(1) the hotspot is represented by an isobaric deuteriumtritium (DT) mixture, with a central hot region at a peak temperature $T_{1}$, surrounded by ten layers, each at decreasing temperature, uniformly sampling the temperature gradient between $T_{1}$ and $1 \mathrm{keV}$ (the latter being the usual limit defining the border of the hotspot). Given the central ion density $n_{1}$, the densities and temperatures of these layers are determined by the isobaric assumption. The mass of the central hot region is assumed to equal the mass of the DT vapour before the implosion (770 ng, for a standard, or Rev. 5, design ${ }^{5}$ ), whilst the mass $m_{h o t}$ of the gradient region-equally distributed between layers - is assumed to be the ablated mass (a few $\mu \mathrm{g}$ ) from the inner wall of the compressed DT ice layer, and it is one of the fit parameters of the model. The mix is modeled as an additional mass $m_{\text {mix }}$ of $\mathrm{Ge}: \mathrm{CH}$ (at $1 \%$ doping for Rev. 5) confined within a circular section of the gradient region of angle $\theta$, at the same temperatures as the corresponding DT, and equally distributed between layers (the mix at the peak temperature $T_{1}$ is assumed by default to be 0 , since the mix will generally be colder than the hottest part of the DT). The function of the angle $\theta$ is to vary the typical photon escape length $L$ (rather than the physical size of the mix jets) and the density $\rho$ of the mix material, so as to mimic opacity effects $(\sim \rho L)$ on the line emission from mix, as discussed later.

(2) the cold DT fuel is modeled as a uniform layer at an ion density $n_{2}$ with a mass given by the total DT ice mass before the implosion (170 $\mu \mathrm{g}$ for Rev. 5) minus $m_{h o t}$, the DT ice contribution to the hotspot. The temperature of this layer does not affect the results significantly, and as such, it is arbitrarily set at $50 \mathrm{eV}$ (warm but ionized hydrogen). The density, however, determines the outer 
radius of the layer and so it affects the curvature of the external shell region, thus affecting the optical thickness of the shell: an example for this effect will be discussed in Sec. IV.

(3) the plastic $(\mathrm{CH})$ ablator is divided into two uniform regions, representing the unablated compressed shell and the ablated low density material. The total mass remaining in the unablated shell $m_{C H}$ is one of the fit parameters of the model, as are its temperature $T_{3}$ and ion density $n_{3}$. This region is responsible for most of the absorption of the X-ray spectra emitted from the central hot region of the target. It is assumed to be uniformly doped with Ge (or other dopants), with a total number of Ge atoms that depend on the design of the dopant layers, and what fraction of such layers are contained within the innermost fraction $m_{C H}$ of the total mass of the shell $(2.6 \mathrm{mg}$ for Rev. 5) before the ablation process. Any remaining $\mathrm{C}, \mathrm{H}$, or dopant ions, obtained by the difference between the ones in the compressed layer and the total number of atoms present in the shell before the implosion, are then used to uniformly populate the outermost layer of the model representing the ablated material. This layer is set to have a temperature of $300 \mathrm{eV}$ and to extend up to a radius $R_{\max }$ (determining its density), which represents the spatial maximum radius of the simulation.

The model just described provides a full self-consistent description of the imploded target, depending on the $10 \mathrm{fit}$ parameters $T_{1}, n_{1}, m_{\text {hot }}, m_{\text {mix }}, \theta, n_{2}, T_{3}, n_{3}, m_{C H}$, and $R_{\text {max }}$. Further to these parameters, an additional choice is required about the continuum lowering model, ${ }^{15}$ which significantly affects the spectroscopic properties of the Ge: $\mathrm{CH}$ shell, as discussed in more detail in Sec. IV B. Once all of these parameters are set, we calculate an emission spectrum by performing radiation transfer with Cretin and by observing the integrated emission intensity over a set of $200 \times 200$ parallel rays in the equatorial direction, sampling the target with $1 \mu \mathrm{m}$ resolution: the resulting space-integrated spectrum is then multiplied by a 125 ps time window (the X-ray burnwidth $^{16}$ ) in order to get an absolute spectral emission to be compared with the experiment.

\section{TEST CASE: HYDRA SIMULATION}

In order to test the predictive capabilities of the model, we first use it to analyse the emission spectra arising from simulated hydrodynamic implosions calculated using the Hydra code. ${ }^{2,17}$ In these simulations, a series of defects on the surface of the capsule seeds Rayleigh-Taylor instabilities that drive jets of $\mathrm{Ge}: \mathrm{CH}$ inside the hotspot, containing an amount of mix that depends on the shape of the given defect. We take a snapshot at the time of peak compression for one of such simulations (with a known amount of mix and known plasma profile) and post-process it with Cretin by using the same ray transfer setup as described in Sec. II, in order to obtain an emission spectrum. We then vary the parameters of our 2D model to fit such a spectrum and compare the resulting mix and plasma parameters with the ones provided by the Hydra simulation. Note that by using the same spectral synthesis procedure for both the Hydra spectrum and the spectrum generated in our 2D model, we perform a test of the predictive capabilities of the fit that is not affected by uncertainties on the physics models used in the codes. In particular, in the following analysis, we use the same continuum lowering model for both spectra, namely, the model by Ecker and Kröll, ${ }^{18}$ which also provides the best fit to the experimental case discussed in Sec. IV.

The spectrum arising from one of the aforementioned Hydra simulations and a fit produced by tuning the ten fit parameters of our 2D model are shown in Fig. 2. It is clear from the figure that, in spite of the relatively simplified geometry of our model, the resulting spectrum is able to capture all of the main spectral features exhibited by the more realistic hydrodynamic simulation. Moreover, the estimated amount of mix from the fit $(18 \mathrm{ng})$ is very close to the real mix mass from Hydra $(21 \mathrm{ng})$, indicating that the model is indeed sensitive to low levels of mix, well below the $75 \mathrm{ng}$ limit.

The ability of such a simplified model to capture all of the spectral features can be understood by relating such features to the different regions within the 2D model. The spectrum in Fig. 2 can be decomposed into a continuum emission component and several bound-bound features. With reference to the region numbering given in Sec. II, the continuum photons are mainly generated in region 1 primarily by the large mass of hot DT but with a non-zero contribution by the hot mix material. However, regions 2 and 3 significantly contribute to the continuum spectrum through opacity effects: the sudden decrease in emission around $10800 \mathrm{eV}$ in the spectrum corresponds to Ge K-shell absorption in the unablated portion of region 3 , where the density and temperature of the Ge:CH shell determine the position and shape of that K-edge feature; the curvature of the continuum observed around $9500 \mathrm{eV}$ is both a function of the plasma conditions of region 3 and a function of its internal radius of curvature, which is determined by the DT density in region 2 . The spectrum also shows four main bound-bound features: the Ge $\mathrm{He}_{\alpha}$ feature (around $10200 \mathrm{eV}$ ) and its red-wing shoulder (around $10100 \mathrm{eV}$ ) are the main indicator of hotspot mix and both arise from the mix wedge in region 1, where the gradient of temperatures within the model allows it to capture the structure of both of these two features at once (in contrast to the previous uniform modelling of the mix material ${ }^{6,10}$ ); a Ge Ly-alpha feature is also observed (around $10600 \mathrm{eV}$ ), whose intensity (relative to the $\mathrm{He}_{\alpha}$ ) depends mainly on the temperature of region 1; finally, a $\mathrm{K}_{\alpha}$ emission/absorption feature (around $9900 \mathrm{eV}$ ) is also observed as a consequence of the K-shell absorption generating the K-edge feature. The $\mathrm{K}_{\alpha}$ bound-bound opacity is strongly determined by the temperature of region 3 and-due to this strong dependence and to the gradient-free nature of the shell in the $2 \mathrm{D}$ model-a compromise between fitting this feature and fitting the $\mathrm{K}$ edge slope is necessary, as is clear from the fit in Fig. 2. Having demonstrated that the model is able to fit the different spectral features, as well as predicting the amount of mix, we turn to considering how the plasma spatial profile returned by the fit compares with the spatial profile given by the hydrodynamic simulation: this is shown in Figs. 3 and 4. 


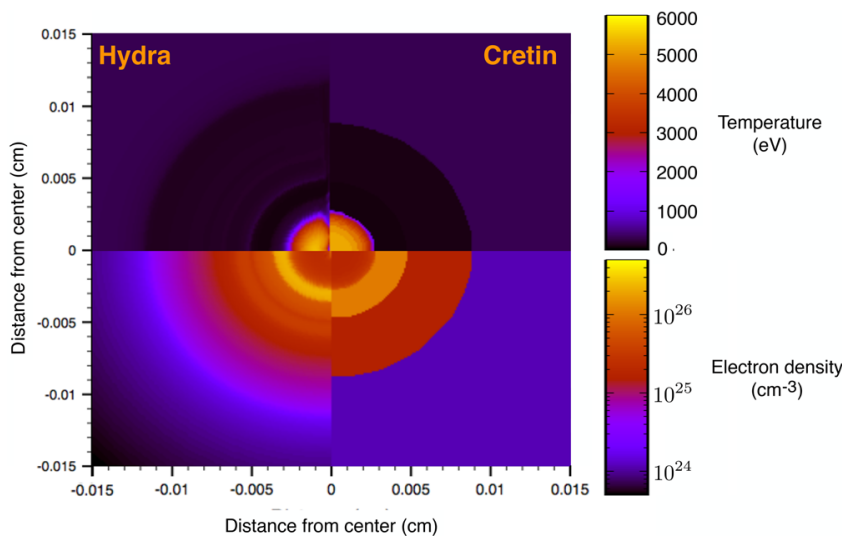

FIG. 3. Comparison between the temperature (top half) and electron density (bottom half) spatial profiles as given by the Hydra simulation (left halfHydra) and as returned by the fit (right half-Cretin). The radii of the different layers in the $2 \mathrm{D}$ model are determined by the densities given by the fit and reproduce well the size of the different zones in the Hydra implosion.

From Fig. 3, it can be deduced that even if the 2D model neglects the density and temperature gradients in both the cold DT layer (region 2) and the ablator layer (region 3)clearly visible in the Hydra simulation-the fit does an excellent job in reproducing both the hotspot region and the average densities of the remaining layers. As the densities are also what determine the size of each layer in the model, this also means that the radii of the uniform layers in the fit closely match the boundaries of the structured cold DT and shell regions in the Hydra simulation. Moreover, if we focus on the profiles for the Ge ion density, as done in Fig. 4, we can see that even if the model is not strictly representative of the Ge distribution in the Hydra case, it is instead quite successful in reproducing the typical (horizontal) photon escape length $(L)$ and typical density $(\rho)$ of the jet, which means that the model is able to capture line-opacity effects $(\sim \rho L)$ for the $\mathrm{He}_{\alpha}$ line emitted by the mix material, as intended.

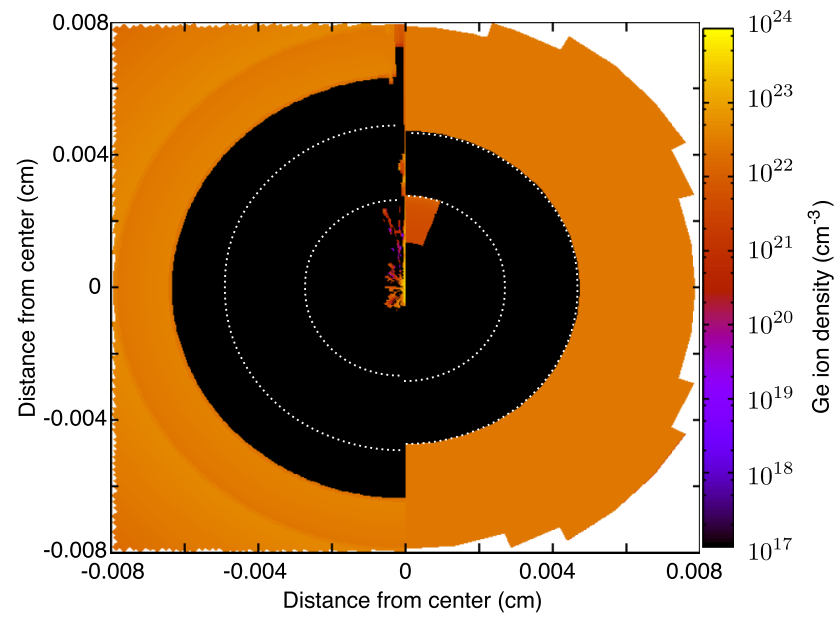

FIG. 4. Comparison between the distribution of the Ge ions in the Hydra simulation (left half) and in the fit case (right half). The white dashed lines are meant to guide the eye to locate the inner/outer surface of the cold DT layer (a small layer of the shell is undoped in the Hydra profile, as by experimental design). The typical density of the jet and the effects of the photon escape length (in the horizontal ray transfer direction) on the mix are well reproduced.
Thus, by testing the model against a (well-characterized) hydrodynamic case, we have demonstrated that it is indeed able to diagnose both the amount of hotspot mix and the radial profile of the imploded target. Although a low mix case has been discussed here to show the ability of the procedure to detect small amounts of mix, the same analysis has also been performed for a hydrodynamic simulation injecting a much more turbulent mix jet of $185 \mathrm{ng}$, distributed in a $\sim 45^{\circ}$ cone. Even in this case, the fit predicts a mix mass of $160 \mathrm{ng}$, a number within $15 \%$ of the correct value, with predicted spatial profiles as accurate as those shown in Figs. 3 and 4.

\section{EXPERIMENTAL CASE AND DISCUSSION}

Having shown that the 2D fit is able to accurately predict all of the main properties of a simulated implosion, we can now apply this tested diagnostic tool to the analysis of experimental data.

In Fig. 5, we plot a previously published experimental spectrum for a NIF implosion of a tri-doped target ${ }^{6}$ (containing $\mathrm{Ge}, \mathrm{Si}$, and $\mathrm{Cu}$ in the ablator). The spectrum differs from the one in Fig. 2 in that the $\mathrm{K}_{\alpha}$ feature is now not reabsorbed by a high-opacity shell and in that a secondary K-edge $+\mathrm{K}_{\alpha}$ structure is observed at low energies due to the $\mathrm{Cu}$ dopants. The figure also shows two slightly different fits to the spectrum, using a version of the 2D model where the fixed quantities (masses, dopant species, and doping levels) are modified according to the experimental design. The fits labelled as simulations 1 and 2 only differ in the estimate of the parameter $m_{\text {hot }}$ (the mass of cold fuel that has been heated to hotspot temperatures, equal to $9 \mu \mathrm{g}$ and $4.5 \mu \mathrm{g}$, respectively), and the fact that simulation 1 , although in good agreement with the overall experimental spectrum, has been slightly biased $(-0.05 \mathrm{~J} / \mathrm{keV} / \mathrm{ster})$, whereas simulation 2 , although within the errorbars without any bias, presents a slightly worse overall agreement. Once again, the 2D model is able to reasonably describe all of the main features of the spectrum, although the comparison shows clear discrepancies in the position and broadening of the emission lines; we attribute these to uncertainties both in the atomic physics

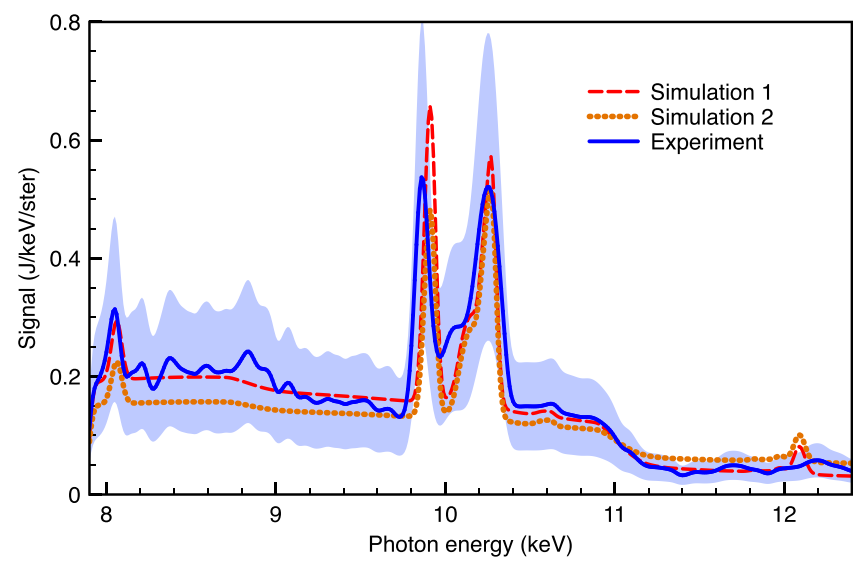

FIG. 5. Experimental spectrum [Regan et al., PRL 111, 045001 (2013)] for an implosion where the ablator contains $\mathrm{Ge}, \mathrm{Si}$, and $\mathrm{Cu}$ dopants, compared with two fits using the 2D model. The shaded band is given by the nominal experimental error $(50 \%)$ of the calibrated spectrometer. 
models (likely inaccurate in the extreme conditions of a NIF implosion) and in the absolute photon energy calibration of the spectrometer.

The fit in Fig. 5 gives an estimate of $35 \mathrm{ng}$ for the amount of mix mass in the implosion, which compares very well with the previous estimate of $34 \mathrm{ng}$ obtained by the analysis of the $\mathrm{He}_{\alpha}$ feature ${ }^{6}$ (in the following, we will refer to any of the work related to such analysis by the label SPR13). However, the current estimate has been performed using a model that enforces self-consistency on all of the properties of the imploded target, and it would thus be reasonable to expect an improvement in the accuracy of the estimate itself, which was limited to $(-13,+50) \mathrm{ng}$ in SPR13. Unfortunately, providing errorbars for the current estimate is a non-trivial issue, due to the large number of fit parameters and to the several-minute calculation time for the 2D model, both preventing an efficient estimation of the fit errors through a standard grid-search procedure (all of the presented fits were instead performed by manually tuning the fit parameters, until a reasonable fit to the spectrum was found). Nevertheless, both qualitative and quantitative discussions on the accuracy of the fit are presented below.

\section{A. Evaluation of the errorbars}

A qualitative estimate of the sensitivity of the fit to the amount of mix can be gleaned from Fig. 6. The figure shows the effects of varying some of the parameters of the fit in Fig. 5 (Simulation 1) on the resulting spectra. As we are interested in knowing whether the experimental spectrum could still be consistent with a mix level as high as the $75 \mathrm{ng}$ limit (the previous errorbar going up to $84 \mathrm{ng}$ ), we increase the amount of mix from 35 up to $75 \mathrm{ng}$. As a consequence, a large difference is observed in the intensity of the $\mathrm{He}_{\alpha}$ emission-as expected-but a small increase is also observed in the level of the continuum. If we then try to compensate for the increased line emission by increasing the opacity of the mix jet (reducing the wedge angle), as can be seen from the figure, we find that any such change would significantly

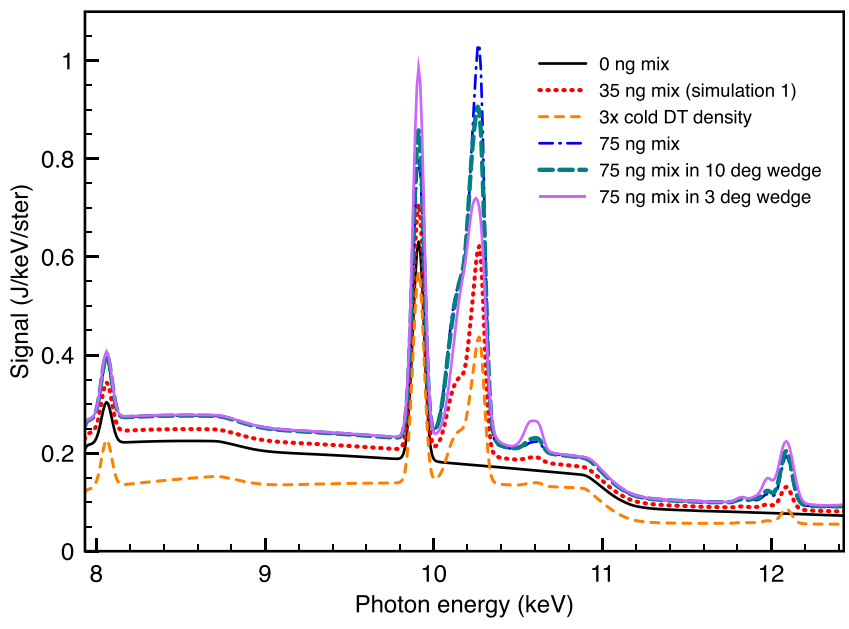

FIG. 6. Variation of the spectra from the $2 \mathrm{D}$ model as a function of the mix mass and opacity effects. All simulations are generated by resetting the parameters as described in the legend, starting from the simulation labelled as Simulation 1 in Fig. 5. affect neither the continuum level nor the red-wing shoulder to the $\mathrm{He}_{\alpha}$, so that consistency with the $75 \mathrm{ng}$ case cannot be achieved this way. On the other hand, if we were to compensate for the line intensity by reducing the density or temperature, the variation in the continuum level would be far more dramatic than those on the line profile, as can be evinced from two facts: first, the main contribution to the continuum level arises from the much larger mass of DT (a few $\mu \mathrm{g}$ ) rather than from the mix, as indicated by the spectrum with $0 \mathrm{ng}$ of mix in the figure (this insensitivity of the continuum to the low level of mix is in agreement with the findings of Ma et al. ${ }^{11}$ ); second, any variation in density on the internal layers forces by self-consistency a variation in the full geometry of the target, affecting the opacity of the shell and changing the overall shape of the spectrum, as observed in the curve corresponding to a three times denser cold DT layer shown in the figure. Moreover, by varying density and temperature, the agreement with all of the line ratios (including the $\mathrm{He}_{\alpha}$ vs red-wing satellites) would equally be lost, and so, this route cannot bring the $75 \mathrm{ng}$ case into agreement with the target spectrum either. In conclusion, the additional constraints provided by our model do indeed improve the accuracy of the mix estimate, with the discussion concerning the results shown in Fig. 6 indicating errorbars which confine the estimate with a central value of $35 \mathrm{ng}$ to values lower than $75 \mathrm{ng}$.

Furthermore, the matters discussed above also show that a 2D self-consistent model is required for a correct estimate of the shell opacity, as simulations with exactly the same shell parameters but different hotspot conditions (Fig. 5) or cold DT conditions (Fig. 6) can produce different shell opacities due to geometric effects, which cannot be determined by modelling the doped ablator independently.

A more quantitative estimate of the errorbars would require the development of a more rigorous automated fit procedure and-due to the difficulties entailed by the large number of fit parameters - this will require a longer term future effort involving the development of efficient computing algorithms. However, a preliminary quantitative analysis has been performed to roughly estimate the typical errorbars achievable by a generic fit procedure using our 2D model. This has been done by randomly sampling the tendimensional space of the 2D simulations with a set of 15000 cases (each case composed of a fixed randomly chosen set of the ten fit parameters and by the corresponding spectrum) and using 1000 of them as test cases. By assuming that a function $f(x)$ exists transforming a given spectrum $x$ uniquely into a vector of ten parameters $\vec{y}$ (those characterizing the 2D model), for each of the 1000 test cases - with spectrum $x_{i}$ - a locally weighted regression procedure is used to fit such a function using the remaining 14000 example cases, resulting in a predicted set of ten parameters for the given test, equal to $\vec{y}_{i}=f_{f i t}\left(x_{i}\right)$ : such predicted parameters are then compared with the actual parameters for the given test case to provide errorbars for the ten quantities of interest, and the procedure is then repeated 1000 times to perform a statistical analysis. The typical (median) errors produced by this study are within a factor of 2 for all the parameters $(\sim 40 \%$ relative error on average) and result in $\sim 80 \%$ relative error for the 


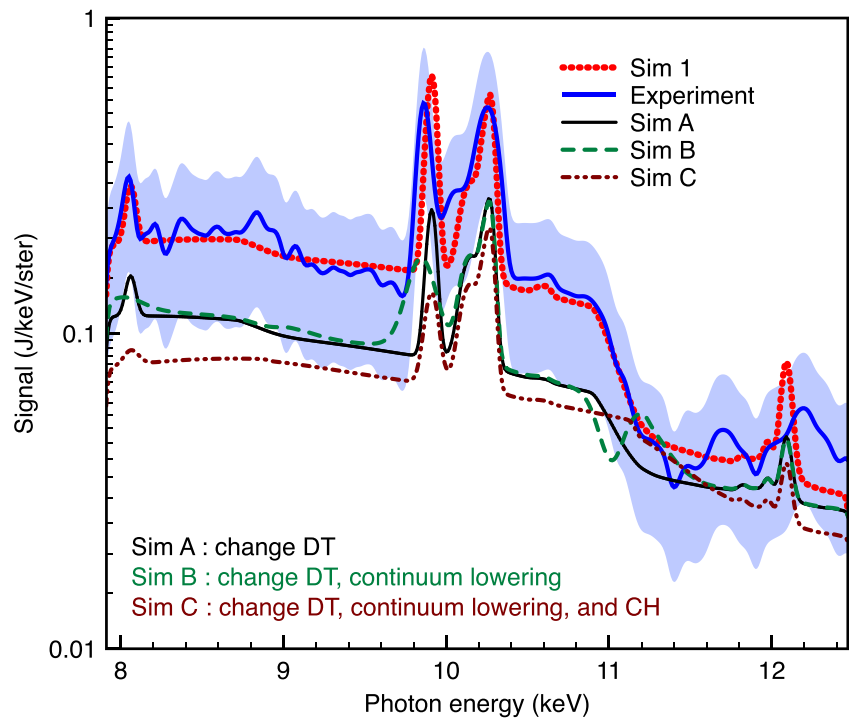

FIG. 7. The fit to the experimental spectrum as in Fig. 5 (Sim 1) is here progressively modified such as follows: first, the hotspot temperature and density are set to the value provided by the old $\mathrm{He}_{\alpha}$ fit in SPR13 ( $\operatorname{Sim} \mathrm{A}$ ), then the continuum lowering model is also changed to match the models (Stewart-Pyatt/ion-sphere) commonly used by the community (Sim B), and finally, the shell density is also readjusted to the value resulting from matching the K-edge feature's position to the predictions of such models (Sim C).

hotspot mix mass. Although this rough estimate can be likely improved by a more accurate automatic fit procedure, such errorbars would already mean that the range of possible mix mass values for the $35 \mathrm{ng}$ case discussed above would not reach the $75 \mathrm{ng}$ limit.

\section{B. Sensitivity to models of continuum lowering}

As a final point in our discussion, we draw attention to a topical issue in dense plasma modelling that also affects the plasma conditions inferred through spectroscopy in a NIF implosion, but the effect of which may be mitigated by the use of self-consistent models such as the one presented here: these are namely, the high-density effects known as continuum lowering and pressure ionization. ${ }^{15}$

In Fig. 7, the fit to the experimental case discussed earlier is progressively modified so that the $2 \mathrm{D}$ model calculation roughly matches the plasma conditions used in SPR13, as well as standard continuum lowering models usually employed by the community. First, the temperature and ion density of the hotspot are modified (Sim A) to match the previously determined values for the $\mathrm{CH}$ :Ge mix material: both parameters are slightly lower than the ones for Simulation 1 (see Table I at the end of this paper for a list of fit parameters for all of the presented simulations), so that the continuum level of the spectrum now drops at the very bottom of the errorbars region (however, the density used only represents an upper limit for the DT in the original modelling, as generally the DT ion density is expected to be even lower than the Ge:CH density, which is what was actually estimated in SPR13).

Although the spectrum for $\operatorname{Sim} \mathrm{A}$ is still within the errorbars and still matches the overall shape of the experimental spectrum, the agreement is quickly lost when a further modification is applied: as previously mentioned, in the current analysis, the Ecker and Kröll model ${ }^{18}$ for continuum lowering was used in order to get the fit Sim 1, whilst the Stewart and Pyatt model ${ }^{19}$ (or equivalently an ion-sphere model ${ }^{15}$ ) used in SPR13 is now used to turn Sim A into Sim B. The effects of the different continuum lowering model are immediately visible in Fig. 7: whilst all of the features arising from the hot parts of the target (the continuum emission level and the $\mathrm{He}_{\alpha}$ feature) are essentially unmodified, the features arising from atomic processes in the ablator layer $(\mathrm{Ge}$ and $\mathrm{Cu} \mathrm{K}$-edges, and $\mathrm{K}_{\alpha}$ lines) now present features that are clearly identifiable as bound-bound processes which are absent in the experimental case. Such features arise from atomic states that are bound at the density of Sim B, according to the Stewart and Pyatt model, whilst they are pushed above the continuum level in Sim A (pressure ionization) by the Ecker and Kröll model. In order to get rid of such states and get a reasonable K-edge position and still preserve the Stewart and Pyatt model, the estimate of the ion density of the ablator needs to be increased by a factor of $\sim 5$ to transform Sim B into Sim C in Fig. 7. However, now that the calculation is performed in a self-consistent fashion that also takes geometry into account, it can be seen from the figure that doing this produces a different shape and emission level for the spectrum, which pushes Sim C outside the errorbars.

Whilst we were unable to find a reasonable fit to the data using the Stewart and Pyatt model, and although the Ecker and Kröll model leads to a better representation of some experimental data in similar plasma conditions to those in the compressed ablator, ${ }^{20}$ we urge caution in inferring any firm conclusions about the two models based solely on the present analysis: first, we are here performing a fit (rather

TABLE I. Parameters of the 2D model for each of the simulations preseted through this paper.

\begin{tabular}{|c|c|c|c|c|c|c|c|c|c|c|}
\hline Simulation & $T_{1}(\mathrm{eV})$ & $n_{1}\left(\mathrm{~cm}^{-3}\right)$ & $m_{\mathrm{hot}}(g)$ & $m_{\operatorname{mix}}(\mathrm{ng})$ & $\theta(\mathrm{deg})$ & $n_{2}\left(\mathrm{~cm}^{-3}\right)$ & $T_{3}(\mathrm{eV})$ & $n_{3}\left(\mathrm{~cm}^{-3}\right)$ & $m_{\mathrm{CH}}(\mathrm{g})$ & $R_{\max }(\mathrm{cm})$ \\
\hline Figure 2 (Cretin) & 5200 & $1.8 \times 10^{25}$ & $1 \times 10^{-5}$ & 18 & 20 & $1.1 \times 10^{25}$ & 80 & $5.8 \times 10^{24}$ & $1.5 \times 10^{-4}$ & 0.055 \\
\hline Figures 5, 6, 7 (Sim 1) & 3800 & $3 \times 10^{24}$ & $9 \times 10^{-6}$ & 35 & 30 & $3 \times 10^{24}$ & 80 & $3 \times 10^{24}$ & $0.5 \times 10^{-3}$ & 0.3 \\
\hline Figure $5(\operatorname{Sim} 2)$ & 3800 & $3 \times 10^{24}$ & $4.5 \times 10^{-6}$ & 35 & 30 & $3 \times 10^{24}$ & 80 & $3 \times 10^{24}$ & $0.5 \times 10^{-3}$ & 0.3 \\
\hline Figure 6 (0 ng) & 3800 & $3 \times 10^{24}$ & $9 \times 10^{-6}$ & 0 & 30 & $3 \times 10^{24}$ & 80 & $3 \times 10^{24}$ & $0.5 \times 10^{-3}$ & 0.3 \\
\hline Figure $6(75 \mathrm{ng})$ & 3800 & $3 \times 10^{24}$ & $9 \times 10^{-6}$ & 75 & 30 & $3 \times 10^{24}$ & 80 & $3 \times 10^{24}$ & $0.5 \times 10^{-3}$ & 0.3 \\
\hline Figure $6(75 \mathrm{ng}, 10 \mathrm{deg})$ & 3800 & $3 \times 10^{24}$ & $9 \times 10^{-6}$ & 75 & 10 & $3 \times 10^{24}$ & 80 & $3 \times 10^{24}$ & $0.5 \times 10^{-3}$ & 0.3 \\
\hline Figure 6 (75 ng, $3 \mathrm{deg})$ & 3800 & $3 \times 10^{24}$ & $9 \times 10^{-6}$ & 75 & 3 & $3 \times 10^{24}$ & 80 & $3 \times 10^{24}$ & $0.5 \times 10^{-3}$ & 0.3 \\
\hline Figure $7(\operatorname{Sim} A, B)$ & 3000 & $2.5 \times 10^{24}$ & $9 \times 10^{-6}$ & 35 & 30 & $3 \times 10^{24}$ & 80 & $3 \times 10^{24}$ & $0.5 \times 10^{-3}$ & 0.3 \\
\hline Figure $7(\operatorname{Sim} C)$ & 3000 & $2.5 \times 10^{24}$ & $9 \times 10^{-6}$ & 35 & 30 & $3 \times 10^{24}$ & 250 & $1.44 \times 10^{25}$ & $0.26 \times 10^{-3}$ & 0.3 \\
\hline
\end{tabular}


than a predictive analysis ${ }^{21}$ ) and any good fit would be by definition consistent with any chosen model; second, a reasonable fit using the Stewart and Pyatt model may still be found, perhaps once the fit procedure has been automatised, making it easier to converge to a best fit for any given model; finally, recent experiments seriously question the applicability itself of such analytical continuum lowering models in atomic kinetics calculations involving mixtures (such as our $\mathrm{Ge}: \mathrm{CH}){ }^{22}$

Nevertheless, the present discussion shows once again that the use of a fully self-consistent model, able to capture all of the spectral features at once, can exclude experimentbased estimates on a given set of plasma conditions ( $\operatorname{Sim~C),~}$ even when such estimates would still be perfectly valid if looking at a subset of features individually. Moreover, if indeed a fit using certain physical models (such as the Stewart and Pyatt one) could not be found (even with a more rigorous fit procedure), such analysis would be extremely useful in discriminating between different models, in a regime of extreme plasma conditions where there is a strong need for model validation.

\section{CONCLUSIONS}

We have discussed a 2D model for the analysis of X-ray emission from a NIF implosion that uses radiation transfer and provides self-consistency between the plasma parameters and the capsule geometry. The simulated spectra are able to describe all of the main spectral features observed in experiments where spectroscopy was used to study the degree of hotspot mix. The model has been tested by fitting simulated spectra from hydrodynamic simulations, producing excellent agreement between the predicted and actual plasma parameters, level of mix and plasma radial profile, which provides confidence in its use as a valid diagnostic.

We used the new tool in order to estimate the amount of mix mass from previously published experimental NIF spectra, ${ }^{6}$ obtaining a nearly identical value to the previous estimate of mix. However, a preliminary analysis for the errorbars indicates that the 2D model can significantly improve the accuracy of spectroscopic estimates of mix, with typical errorbars below the radiative cooling limit of $75 \mathrm{ng}$ for low levels of mix. This is achieved by the increased number of constraints in fitting the data, including geometric and design constraints as well as the larger number of spectral features used; in particular, we have shown that the spectra are very sensitive to opacity changes in the compressed ablator region, which are affected-through selfconsistency - by the density of the innermost layers (changing their in-flight aspect ratio).

Future work will include the development of a fully automated fit procedure, involving the development of efficient computing algorithms able to handle the large number of fit parameters. This is needed in order to improve the estimate of the errorbars on a given fit, as well as to guarantee the convergence to a best fit for the estimate of experimental quantities.

Further to the analysis of mix spectroscopy data, the model can be easily applied to the study of more general implosions, including the continuum spectra analysis of NIF shots without Ge dopants, or the design of non-ignition experiments such as self-radiography implosions. ${ }^{12}$

\section{ACKNOWLEDGMENTS}

O.C. wishes to thank Prav Patel and Tammy Ma for useful discussions. O.C., P.D., J.S.W. and N.C.W. are grateful to EPSRC for support under the linked Grant Nos. EP/L000849/1 and EP/L000644/1, and O.C. J.S.W. and T.R.P. also received support under Grant No. EP/H035877/ 1. S.M.V. would like to acknowledge funding from the Royal Society. S.P.R. and R.E. received support for this work from the Department of Energy National Nuclear Security Administration under Award No. DE-NA0001944, the University of Rochester, and the New York State Energy Research and Development Authority. The work of H.S. and B.H. was performed under the auspices of the U.S. Department of Energy by Lawrence Livermore National Laboratory under Contract No. DE-AC52-07NA27344.

Due to the confidential nature of some of the research materials supporting this publication not all of the data can be made accessible to other researchers. Please contact the corresponding author for more information.

${ }^{1}$ J. D. Lindl, P. Amendt, R. L. Berger, S. G. Glendinning, S. H. Glenzer, S. W. Haan, R. L. Kauffman, O. L. Landen, and L. J. Suter, Phys. Plasmas 11, 339 (2004)

${ }^{2}$ B. Hammel, S. Haan, D. Clark, M. Edwards, S. Langer, M. Marinak, M. Patel, J. Salmonson, and H. Scott, High Energy Density Phys. 6, 171 (2010); in Proceedings of the 2nd International Conference on High Energy Density Physics (ICHED) (2009).

${ }^{3}$ J. D. Lindl and E. I. Moses, Phys. Plasmas 18, 050901 (2011).

${ }^{4}$ S. H. Glenzer, D. A. Callahan, A. J. MacKinnon, J. L. Kline, G. Grim, E. T. Alger, R. L. Berger, L. A. Bernstein, R. Betti, D. L. Bleuel, T. R. Boehly, D. K. Bradley, S. C. Burkhart, R. Burr, J. A. Caggiano, C. Castro, D. T. Casey, C. Choate, D. S. Clark, P. Celliers, C. J. Cerjan, G. W. Collins, E. L. Dewald, P. DiNicola, J. M. DiNicola, L. Divol, S. Dixit, T. Dppner, R. Dylla-Spears, E. Dzenitis, M. Eckart, G. Erbert, D. Farley, J. Fair, D. Fittinghoff, M. Frank, L. J. A. Frenje, S. Friedrich, D. T. Casey, M. G. Johnson, C. Gibson, E. Giraldez, V. Glebov, S. Glenn, N. Guler, S. W. Haan, B. J. Haid, B. A. Hammel, A. V. Hamza, C. A. Haynam, G. M. Heestand, M. Hermann, H. W. Hermann, D. G. Hicks, D. E. Hinkel, J. P. Holder, D. M. Holunda, J. B. Horner, W. W. Hsing, H. Huang, N. Izumi, M. Jackson, O. S. Jones, D. H. Kalantar, R. Kauffman, J. D. Kilkenny, R. K. Kirkwood, J. Klingmann, T. Kohut, J. P. Knauer, J. A. Koch, B. Kozioziemki, G. A. Kyrala, A. L. Kritcher, J. Kroll, K. L. Fortune, L. Lagin, O. L. Landen, D. W. Larson, D. LaTray, R. J. Leeper, S. L. Pape, J. D. Lindl, R. Lowe-Webb, T. Ma, J. McNaney, A. G. MacPhee, T. N. Malsbury, E. Mapoles, C. D. Marshall, N. B. Meezan, F. Merrill, P. Michel, J. D. Moody, A. S. Moore, M. Moran, K. A. Moreno, D. H Munro, B. R. Nathan, A. Nikroo, R. E. Olson, C. D. Orth, A. E. Pak, P. K. Patel, T. Parham, R. Petrasso, J. E. Ralph, H. Rinderknecht, S. P. Regan, H. F. Robey, J. S. Ross, M. D. Rosen, R. Sacks, J. D. Salmonson, R. Saunders, J. Sater, C. Sangster, M. B. Schneider, F. H. Sguin, M. J. Shaw, B. K. Spears, P. T. Springer, W. Stoeffl, L. J. Suter, C. A. Thomas, R. Tommasini, R. P. J. Town, C. Walters, S. Weaver, S. V. Weber, P. J. Wegner, P. K. Whitman, K. Widmann, C. C. Widmayer, C. H. Wilde, D. C. Wilson, B. V. Wonterghem, B. J. MacGowan, L. J. Atherton, M. J. Edwards, and E. I. Moses, Phys. Plasmas 19, 056318 (2012).

${ }^{5}$ S. W. Haan, J. D. Lindl, D. A. Callahan, D. S. Clark, J. D. Salmonson, B. A. Hammel, L. J. Atherton, R. C. Cook, M. J. Edwards, S. Glenzer, A. V. Hamza, S. P. Hatchett, M. C. Herrmann, D. E. Hinkel, D. D. Ho, H. Huang, O. S. Jones, J. Kline, G. Kyrala, O. L. Landen, B. J. MacGowan, M. M. Marinak, D. D. Meyerhofer, J. L. Milovich, K. A. Moreno, E. I. Moses, D. H. Munro, A. Nikroo, R. E. Olson, K. Peterson, S. M. Pollaine, J. E. Ralph, H. F. Robey, B. K. Spears, P. T. Springer, L. J. Suter, C. A. 
Thomas, R. P. Town, R. Vesey, S. V. Weber, H. L. Wilkens, and D. C. Wilson, Phys. Plasmas 18, 051001 (2011).

${ }^{6}$ S. P. Regan, R. Epstein, B. A. Hammel, L. J. Suter, H. A. Scott, M. A. Barrios, D. K. Bradley, D. A. Callahan, C. Cerjan, G. W. Collins, S. N. Dixit, T. Döppner, M. J. Edwards, D. R. Farley, K. B. Fournier, S. Glenn, S. H. Glenzer, I. E. Golovkin, S. W. Haan, A. Hamza, D. G. Hicks, N. Izumi, O. S. Jones, J. D. Kilkenny, J. L. Kline, G. A. Kyrala, O. L. Landen, T. Ma, J. J. MacFarlane, A. J. MacKinnon, R. C. Mancini, R. L. McCrory, N. B. Meezan, D. D. Meyerhofer, A. Nikroo, H.-S. Park, J. Ralph, B. A. Remington, T. C. Sangster, V. A. Smalyuk, P. T. Springer, and R. P. J. Town, Phys. Rev. Lett. 111, 045001 (2013).

${ }^{7}$ R. C. Mancini and C. F. Hooper, Jr., "Hydrodynamics and k-shell radiation emission of laser compressed ar-filled glass microballoons," J. Phys. D: Appl. Phys. 21(7), 1099 (1988).

${ }^{8}$ B. Yaakobi, D. Steel, E. Thorsos, A. Hauer, and B. Perry, "Direct measurement of compression of laser-imploded targets using X-ray spectroscopy," Phys. Rev. Lett. 39, 1526-1529 (1977).

${ }^{9}$ B. Yaakobi, S. Skupsky, R. L. McCrory, C. F. Hooper, H. Deckman, P. Bourke, and J. M. Soures, "High temperature plasma physics and its applications-X-ray spectroscopy of laser imploded targets," Philos. Trans. R. Soc. London, Ser. A 300, 623-630 (1981).

${ }^{10}$ S. P. Regan, R. Epstein, B. A. Hammel, L. J. Suter, J. Ralph, H. Scott, M. A. Barrios, D. K. Bradley, D. A. Callahan, C. Cerjan, G. W. Collins, S. N. Dixit, T. Doeppner, M. J. Edwards, D. R. Farley, S. Glenn, S. H. Glenzer, I. E. Golovkin, S. W. Haan, A. Hamza, D. G. Hicks, N. Izumi, J. D. Kilkenny, J. L. Kline, G. A. Kyrala, O. L. Landen, T. Ma, J. J. MacFarlane, R. C. Mancini, R. L. McCrory, N. B. Meezan, D. D. Meyerhofer, A. Nikroo, K. J. Peterson, T. C. Sangster, P. Springer, and R. P. J. Town, Phys. Plasmas 19, 056307 (2012).

${ }^{11}$ T. Ma, P. K. Patel, N. Izumi, P. T. Springer, M. H. Key, L. J. Atherton, L. R. Benedetti, D. K. Bradley, D. A. Callahan, P. M. Celliers, C. J. Cerjan, D. S. Clark, E. L. Dewald, S. N. Dixit, T. Döppner, D. H. Edgell, R. Epstein, S. Glenn, G. Grim, S. W. Haan, B. A. Hammel, D. Hicks, W. W. Hsing, O. S. Jones, S. F. Khan, J. D. Kilkenny, J. L. Kline, G. A. Kyrala, O. L. Landen, S. Le Pape, B. J. MacGowan, A. J. Mackinnon, A. G. MacPhee, N. B. Meezan, J. D. Moody, A. Pak, T. Parham, H.-S. Park, J. E. Ralph, S. P. Regan, B. A. Remington, H. F. Robey, J. S. Ross, B. K.
Spears, V. Smalyuk, L. J. Suter, R. Tommasini, R. P. Town, S. V. Weber, J. D. Lindl, M. J. Edwards, S. H. Glenzer, and E. I. Moses, Phys. Rev. Lett. 111, 085004 (2013).

${ }^{12}$ L. A. Pickworth, B. A. Hammel, V. A. Smalyuk, A. G. MacPhee, H. A. Scott, H. F. Robey, O. L. Landen, M. A. Barrios, S. P. Regan, M. B. Schneider, M. Hoppe, T. Kohut, D. Holunga, C. Walters, B. Haid, and M. Dayton, Phys. Rev. Lett. 117, 035001 (2016).

${ }^{13}$ H. A. Scott, "Cretin - a radiative transfer capability for laboratory plasmas," J. Quant. Spectrosc. Radiat. Transfer 71, 689 (2001).

${ }^{14}$ H. A. Scott and R. W. Mayle, Appl. Phys. B 58, 35 (1994).

${ }^{15}$ G. Zimmerman and R. More, J. Quant. Spectrosc. Radiat. Transfer 23, 517 (1980).

${ }^{16}$ D. H. Edgell, D. K. Bradley, E. J. Bond, S. Burns, D. A. Callahan, J. Celeste, M. J. Eckart, V. Y. Glebov, D. S. Hey, G. Lacaille, J. D. Kilkenny, J. Kimbrough, A. J. Mackinnon, J. Magoon, J. Parker, T. C. Sangster, M. J. Shoup III, C. Stoeckl, T. Thomas, and A. MacPhee, Rev. Sci. Instrum. 83, 10E119 (2012).

${ }^{17}$ M. M. Marinak, S. W. Haan, T. R. Dittrich, R. E. Tipton, and G. B. Zimmerman, Phys. Plasmas 5, 1125 (1998).

${ }^{18}$ G. Ecker and W. Kröll, Phys. Fluids 6, 62 (1963).

${ }^{19}$ J. C. Stewart and K. D. Pyatt, Jr., Astrophys. J. 144, 1203 (1966).

${ }^{20}$ O. Ciricosta, S. M. Vinko, H.-K. Chung, B.-I. Cho, C. R. D. Brown, T. Burian, J. Chalupský, K. Engelhorn, R. W. Falcone, C. Graves, V. Hájková, A. Higginbotham, L. Juha, J. Krzywinski, H. J. Lee, M. Messerschmidt, C. D. Murphy, Y. Ping, D. S. Rackstraw, A. Scherz, W. Schlotter, S. Toleikis, J. J. Turner, L. Vysin, T. Wang, B. Wu, U. Zastrau, D. Zhu, R. W. Lee, P. Heimann, B. Nagler, and J. S. Wark, Phys. Rev. Lett. 109, 065002 (2012).

${ }^{21}$ O. Ciricosta, S. M. Vinko, H.-K. Chung, C. Jackson, R. W. Lee, T. R. Preston, D. S. Rackstraw, and J. S. Wark, Phys. Plasmas 23, 022707 (2016).

${ }^{22}$ O. Ciricosta, S. M. Vinko, B. Barbrel, D. S. Rackstraw, T. R. Preston, T. Burian, J. Chalupský, B. I. Cho, H.-K. Chung, G. L. Dakovski, K. Engelhorn, V. Hájková, P. Heimann, M. Holmes, L. Juha, J. Krzywinski, R. W. Lee, S. Toleikis, J. J. Turner, U. Zastrau, and J. S. Wark, Nat. Commun. 7, 11713 EP (2016), article. 